Article

\title{
Active Components with Inhibitory Activities on IFN- $\gamma /$ STAT1 and IL-6/STAT3 Signaling Pathways from Caulis Trachelospermi
}

Xiao-Ting Liu ${ }^{1,2}$, Zhe-Xing Wang ${ }^{1}$, Yu Yang ${ }^{2}$, Lin Wang ${ }^{2}$, Ruo-Feng Sun ${ }^{2}$, Yi-Min Zhao ${ }^{2}$ and Neng-Jiang Yu ${ }^{2, *}$

1 School of Traditional Chinese Materia Medica, Shenyang Pharmaceutical University, Shenyang 110016, China

2 Beijing Institute of Pharmacology and Toxicology, Beijing 100850, China

* Author to whom correspondence should be addressed; E-Mail: ynj08@hotmail.com; Tel.: +86-10-6687-4617; Fax: +86-10-6821-1656.

Received: 26 June 2014; in revised form: 25 July 2014 / Accepted: 27 July 2014 /

Published: 5 August 2014

\begin{abstract}
Initial investigation for new active herbal extract with inhibiting activity on JAK/STAT signaling pathway revealed that the extract of Caulis Trachelospermi, which was separated by $80 \%$ alcohol extraction and subsequent HP-20 macroporous resin column chromatography, was founded to strongly inhibit IFN- $\gamma$-induced STAT1-responsive luciferase activity (IFN- $\gamma / \mathrm{STAT} 1$ ) with $\mathrm{IC}_{50}$ value of $2.43 \mu \mathrm{g} / \mathrm{mL}$ as well as inhibiting IL-6-induced STAT3-responsive luciferase activity (IL-6/STAT3) with $\mathrm{IC}_{50}$ value of $1.38 \mu \mathrm{g} / \mathrm{mL}$. Subsequent study on its active components led to the isolation and identification of two new dibenzylbutyrolactone lignans named 4-demethyltraxillaside (1) and nortrachelogenin $4-O-\beta$-D-glucopyranoside (2), together with six known compounds. The lignan compounds 1-4 together with other lignan compounds isolated in previous study were tested the activities on IFN- $\gamma /$ STAT1 and IL-6/STAT3 pathways. The following result showed that the main components trachelogenin and arctigenin had corresponding activities on IFN- $\gamma /$ STAT1 pathway with $\mathrm{IC}_{50}$ values of $3.14 \mu \mathrm{M}$ and $9.46 \mu \mathrm{M}$ as well as trachelogenin, arctigenin and matairesinol strongly inhibiting IL-6/STAT3 pathway with $\mathrm{IC}_{50}$ values of $3.63 \mu \mathrm{M}, 6.47 \mu \mathrm{M}$ and $2.92 \mu \mathrm{M}$, respectively.
\end{abstract}

Keywords: Caulis Trachelospermi; 4-demethyltraxillaside; nortrachelogenin 4-O- $\beta$-Dglucopyranoside; JAK/STAT signaling pathway 


\section{Introduction}

Caulis Trachelospermi, the stems and leaves of Trachelospermum jasminoides (Lindl.) Lem, is mainly distributed in Henan, Anhui, Hubei, Shandong and Guangxi provinces in China. It has been used in Traditional Chinese Medicine for the treatment of rheumatic arthralgia, aching of the loins and knees, traumatic injuries [1] and its medicinal values such as anticancer, anti-inflammation had been reported [2,3]. Chemical investigations indicated that it mainly contained lignans, flavonoids and triterpenoids [4-6]. In our previous study, the extract of Caulis Trachelospermi, which was separated by $80 \%$ alcohol extraction and subsequent HP-20 macroporous resin column chromatography, exhibited marked anti-inflammatory activity in animal model [7] as well as moderate inhibiting activity on NF- $\kappa \mathrm{B}$ signaling pathway induced by TNF- $\alpha$ [8], its active chemical investigation led to the discovery of 30 compounds including 19 dibenzylbutyrolactone lignans [7-11] and its main components, trachelogenin, nortrachelogenin and matairesinol, showed corresponding moderate inhibiting activities on TNF- $\alpha / \mathrm{NF}-\kappa \mathrm{B}$ pathway [8]. The JAK (Janus kinase)/STAT (signal transducer and activator of transcription) pathway is another signaling pathway responsible for signal transduction of a large number of cytokines [12]. Constitutive activation of the JAK/STAT pathway is frequently associated with cancer and autoimmune diseases, and it has been considered as an important target for therapeutic intervention [13-15]. Our recent study revealed the extract of Caulis Trachelospermi and its main components trachelogenin, arctigenin and matairesinol having strong inhibiting activity on the pathway, which may be an imprtant mechanism for the anticancer and anti-inflammation function of Caulis Trachelospermi. To further investigate active components, a phytochemical study guided by HPLC finger print chromatography [16] was performed and two new dibenzylbutyrolactone lignans named 4-demethyltraxillaside (1) and nortrachelogenin 4- $O-\beta$-D-glucopyranoside (2), together with six known compounds (Figure 1) were isolated. Further screening for active compounds resulted that three main dibenzylbutyrolatone lignan components trachelogenin, actigenin and matairesinol had corresponding strong activities on the JAK/STAT pathway. In this paper, we report the isolation and structural elucidation of compounds $\mathbf{1}$ and $\mathbf{2}$ as well as the JAK/STAT pathway inhibiting activities of the extract and lignan components. Spectral data of the known compounds $\mathbf{3}$ and $\mathbf{4}$ whose detailed NMR data has not been reported to date is also described.

Figure 1. Chemical structures of compounds 1-8.

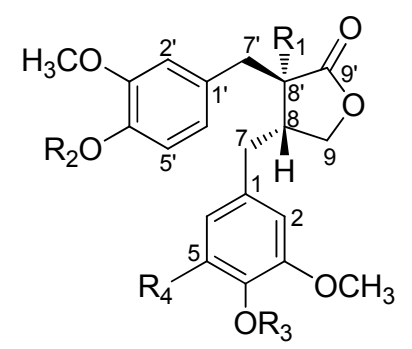

$1 \mathrm{R}_{1}=\mathrm{H} \quad \mathrm{R}_{2}=\mathrm{Glc} \quad \mathrm{R}_{3}=\mathrm{H} \quad \mathrm{R}_{4}=\mathrm{OCH}_{3}$

$2 \mathrm{R}_{1}=\mathrm{OH} \quad \mathrm{R}_{2}=\mathrm{H} \quad \mathrm{R}_{3}=\mathrm{Glc} \mathrm{R}_{4}=\mathrm{H}$

$3 \mathrm{R}_{1}=\mathrm{H} \quad \mathrm{R}_{2}=\mathrm{H} \quad \mathrm{R}_{3}=\mathrm{Glc} \quad \mathrm{R}_{4}=\mathrm{H}$

$4 \mathrm{R}_{1}=\mathrm{OH} \mathrm{R}_{2}=\mathrm{Glc} \mathrm{R}_{3}=\mathrm{Glc} \mathrm{R}_{4}=\mathrm{H}$

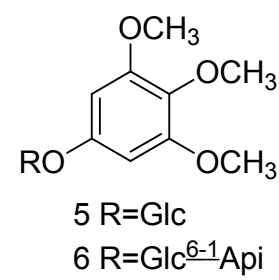

$6 \mathrm{R}=\mathrm{Glc} \stackrel{6-1}{ } \mathrm{Api}$

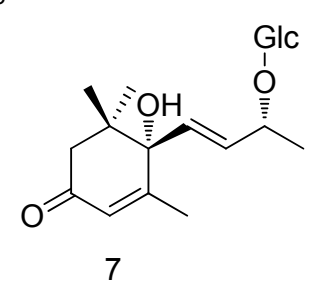

7

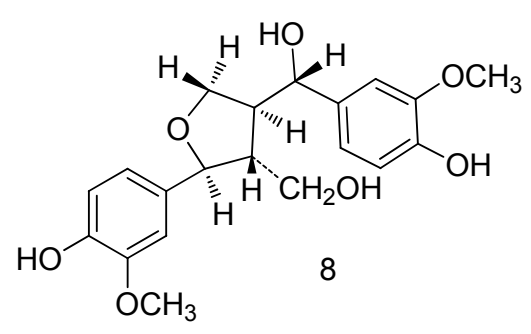




\section{Results and Discussion}

An $80 \%$ alcohol extract of Caulis Trachelospermi was applied on a HP-20 macroporous resin column and eluted with water and 70\% alcohol successively. The $70 \%$ alcohol elution was subjected to multiple chromatographic techniques using Sephadex LH-20, ODS column chromatography (CC) and semi-preparative RP-HPLC to furnish two new lignans and six known compounds.

Compound 1 was obtained as white amorphous powder with the molecular formula $\mathrm{C}_{27} \mathrm{H}_{34} \mathrm{O}_{12}$ on the basis of HRESIMS ( $\mathrm{m} / \mathrm{z} 573.1945[\mathrm{M}+\mathrm{Na}]^{+}$, calcd. 573.1942). ${ }^{1} \mathrm{H}-\mathrm{NMR}$ (Table 1) spectrum displayed signals for an ABX system assignable to 3,4-disubstituted phenyl group at $\delta 6.99(1 \mathrm{H}$, d, $\left.J=8.3 \mathrm{~Hz}, \mathrm{H}-5^{\prime}\right), 6.68\left(1 \mathrm{H}, \mathrm{dd}, J=8.3,1.8 \mathrm{~Hz}, \mathrm{H}-6^{\prime}\right)$ and $6.80\left(1 \mathrm{H}, \mathrm{d}, J=1.8 \mathrm{~Hz}, \mathrm{H}-2^{\prime}\right)$, a singlet at $\delta 6.34$ (2H, s, H-2 and H-6) to present a 3,4,5-trisubstituted phenyl group, an oxygenated methylene at $\delta 4.11(1 \mathrm{H}, \mathrm{dd}, J=8.5,7.2 \mathrm{~Hz}, \mathrm{Ha}-9)$ and $3.89(1 \mathrm{H}, \mathrm{t}, J=8.5 \mathrm{~Hz}, \mathrm{Hb}-9)$, two methylenes at $\delta$ 2.45-2.52 (2H, m, H-7) and 2.80-2.83 (2H, m, H-7'), two methines at $\delta 2.40-2.45(1 \mathrm{H}, \mathrm{m}, \mathrm{H}-8)$ and $2.75\left(1 \mathrm{H}, \mathrm{dd}, J=8.2,6.1 \mathrm{~Hz}, \mathrm{H}-8^{\prime}\right) .{ }^{13} \mathrm{C}-\mathrm{NMR}$ spectrum which was sorted by HMQC data showed the presence of a carbonyl carbon, twelve aromatic carbons including seven quaternary carbons and five tertiary carbons, an oxygenated methylene carbon, four high-field carbons including two methylene carbons and two methine carbons. The spectral data mentioned above is characteristic to dibenzylbutyrolactone lignan. HMBC correlations from C-9' $(\delta 178.6)$ to $\mathrm{H}-7$ ' as well as that from $\mathrm{H}-7^{\prime}$ to three aromatic carbons at $\delta 131.9\left(\mathrm{C}-1^{\prime}\right), 113.9\left(\mathrm{C}-2^{\prime}\right), 121.4\left(\mathrm{C}-6^{\prime}\right)$ indicated that the 3,4-disubstituted phenyl group was connected with C-7'. So, the 3,4,5-trisubstituted phenyl group was deduced the linkage with C-7 which was also proved by HMBC correlations as shown in Figure 2. Carbon signals at $\delta 131.9,113.9,148.7,145.4,115.1,121.4$ were assigned to $\mathrm{C}-1^{\prime}-6$ ' as well as signals at $\delta 128.8$, 106.0, 148.0, 133.9, 148.0, 106.0 were assigned to C-1-6. The ${ }^{1} \mathrm{H}-\mathrm{NMR}$ spectrum also showed three methoxyl groups at $\delta 3.71$ and 3.72 which exhibited respective $\mathrm{HMBC}$ correlations with carbons at $\delta$ 148.0 (C-3, C-5) and 148.7 (C-3'), a phenolic hydroxy at $\delta 8.17(1 \mathrm{H}, \mathrm{s}, 4-\mathrm{OH})$, together with the presence of a sugar anomeric proton at $\delta 4.84(1 \mathrm{H}, \mathrm{d}, J=7.3 \mathrm{~Hz})$. Accordingly, Compound 1 was considered to be the glycoside of 4-demethyltraxillagenin which had been isolated from Caulis Trachelospermi [9]. HMBC correlation between the sugar anomeric hydrogen signal ( $\delta$ 4.84) and C-4' $(\delta$ 145.4) implied that the sugar moiety was connected to C-4'. The diagnostic ${ }^{13} \mathrm{C}-\mathrm{NMR}$ spectral data of the sugar moiety and coupling constant of the anomeric proton $(J=7.3 \mathrm{~Hz})$ indicated the sugar moiety was $\beta$-D-glucopyranosyl [4,17]. Its identical carbon chemical shifts with those of 4-demethyltraxillagenin indicated its absolute configuration was $8 R, 8^{\prime} R$ or $8 S, 8^{\prime} S$. Compound 1 had negative cotton effects at $233(\Delta \varepsilon-2.72)$ and $276 \mathrm{~nm}(\Delta \varepsilon-0.58)$ in $C D$ spectrum, which determined its $8 R, 8^{\prime} R$ configuration according to the report that $8 R, 8^{\prime} R$-isomer has negative cotton effects at 233 and $276 \mathrm{~nm}$ in contrast to the positive cotton effects of $8 S, 8^{\prime} S$-isomer [18]. Consequently, the structure of 1 was identified as $\left(8 R, 8^{\prime} R\right)$-4-hydroxy-3,3',4-trimethoxylignan-9,9'-olide-4'- $O$ - $\beta$-D-glucopyranoside, namely 4-demethyltraxillaside. 
Table 1. ${ }^{1} \mathrm{H}-\mathrm{NMR}(400 \mathrm{MHz})$ and ${ }^{13} \mathrm{C}-\mathrm{NMR}(100 \mathrm{MHz})$ spectral data of compounds $\mathbf{1}$ and 2 (in DMSO- $d_{6}, \delta$ in ppm, $J$ in $\mathrm{Hz}$ ).

\begin{tabular}{|c|c|c|c|c|}
\hline \multirow{2}{*}{ Position } & \multicolumn{2}{|r|}{1} & \multicolumn{2}{|r|}{2} \\
\hline & $\delta_{\mathrm{C}}$ & $\delta_{\mathrm{H}}$ & $\delta_{\mathrm{C}}$ & $\delta_{\mathrm{H}}$ \\
\hline 1 & 128.8 & & 133.0 & \\
\hline 2 & 106.0 & $6.34(1 \mathrm{H}, \mathrm{s})$ & 113.0 & $6.70(1 \mathrm{H}, \mathrm{d}, 1.6)$ \\
\hline 3 & 148.0 & & 148.8 & \\
\hline 4 & 133.9 & & 145.1 & \\
\hline 5 & 148.0 & & 115.3 & $6.97(1 \mathrm{H}, \mathrm{d}, 8.3)$ \\
\hline 6 & 106.0 & $6.34(1 \mathrm{H}, \mathrm{s})$ & 120.5 & $6.61(1 \mathrm{H}$, br d, 8.3) \\
\hline 7 & 37.3 & $2.45-2.52(2 \mathrm{H}, \mathrm{m})$ & 30.9 & $\begin{array}{c}2.61(1 \mathrm{H}, \mathrm{dd}, 12.3,2.4) \\
2.44(1 \mathrm{H}, \mathrm{br} \mathrm{d}, 12.3)\end{array}$ \\
\hline 8 & 40.9 & $2.40-2.45(1 \mathrm{H}, \mathrm{m})$ & 42.8 & $2.39(1 \mathrm{H}, \mathrm{m})$ \\
\hline 9 & 70.8 & $\begin{array}{c}4.11(1 \mathrm{H}, \mathrm{dd}, 8.5,7.2) \\
3.89(1 \mathrm{H}, \mathrm{t}, 8.5)\end{array}$ & 70.0 & $3.95(2 \mathrm{H}, \mathrm{d}, 7.7)$ \\
\hline $1^{\prime}$ & 131.9 & & 126.4 & \\
\hline $2^{\prime}$ & 113.9 & $6.80(1 \mathrm{H}, \mathrm{d}, 1.8)$ & 114.5 & $6.77(1 \mathrm{H}$, br s $)$ \\
\hline $3^{\prime}$ & 148.7 & & 147.2 & \\
\hline $4^{\prime}$ & 145.4 & & 145.4 & \\
\hline $5^{\prime}$ & 115.1 & $6.99(1 \mathrm{H}, \mathrm{d}, 8.3)$ & 115.3 & $6.68(1 \mathrm{H}, \mathrm{d}, 8.0)$ \\
\hline $6^{\prime}$ & 121.4 & $6.68(1 \mathrm{H}, \mathrm{dd}, 8.3,1.8)$ & 122.7 & $6.61(1 \mathrm{H}$, br d, 8.0$)$ \\
\hline $7^{\prime}$ & 33.6 & $2.80-2.83(2 \mathrm{H}, \mathrm{m})$ & $\begin{array}{c}40.0 \\
\text { (overlapped) }\end{array}$ & $\begin{array}{l}2.98(1 \mathrm{H}, \mathrm{d}, 13.8) \\
2.83(1 \mathrm{H}, \mathrm{d}, 13.8)\end{array}$ \\
\hline $8^{\prime}$ & 45.6 & $2.75(1 \mathrm{H}, \mathrm{dd}, 8.2,6.1)$ & 75.4 & \\
\hline $9^{\prime}$ & 178.6 & & 178.1 & \\
\hline $1 "$ & 100.3 & $4.84(1 \mathrm{H}, \mathrm{d}, 7.3)$ & 100.2 & $4.82(1 \mathrm{H}, \mathrm{d}, 7.2)$ \\
\hline $2^{\prime \prime}$ & 73.3 & & 73.2 & \\
\hline $3^{\prime \prime}$ & 77.0 & & 77.0 & \\
\hline $4 "$ & 69.7 & & 69.7 & \\
\hline $5^{\prime \prime}$ & 76.9 & & 76.9 & \\
\hline 6" & 60.7 & & 60.7 & \\
\hline $4-\mathrm{OH}$ & & $8.17(1 \mathrm{H}, \mathrm{s})$ & & \\
\hline 4'-OH & & & & $8.85(1 \mathrm{H}, \mathrm{s})$ \\
\hline 8'-OH & & & & $6.21(1 \mathrm{H}, \mathrm{s})$ \\
\hline $3-\mathrm{OCH}_{3}$ & 56.0 & $3.71(3 \mathrm{H}, \mathrm{s})$ & 55.6 & $3.70(3 \mathrm{H}, \mathrm{s})$ \\
\hline 3'- $-\mathrm{OCH}_{3}$ & 55.7 & $3.72(3 \mathrm{H}, \mathrm{s})$ & 55.6 & $3.73(3 \mathrm{H}, \mathrm{s})$ \\
\hline $5-\mathrm{OCH}_{3}$ & 56.0 & $3.71(3 \mathrm{H}, \mathrm{s})$ & & \\
\hline
\end{tabular}

Figure 2. Key HMBC correlations of compounds 1 and 2.
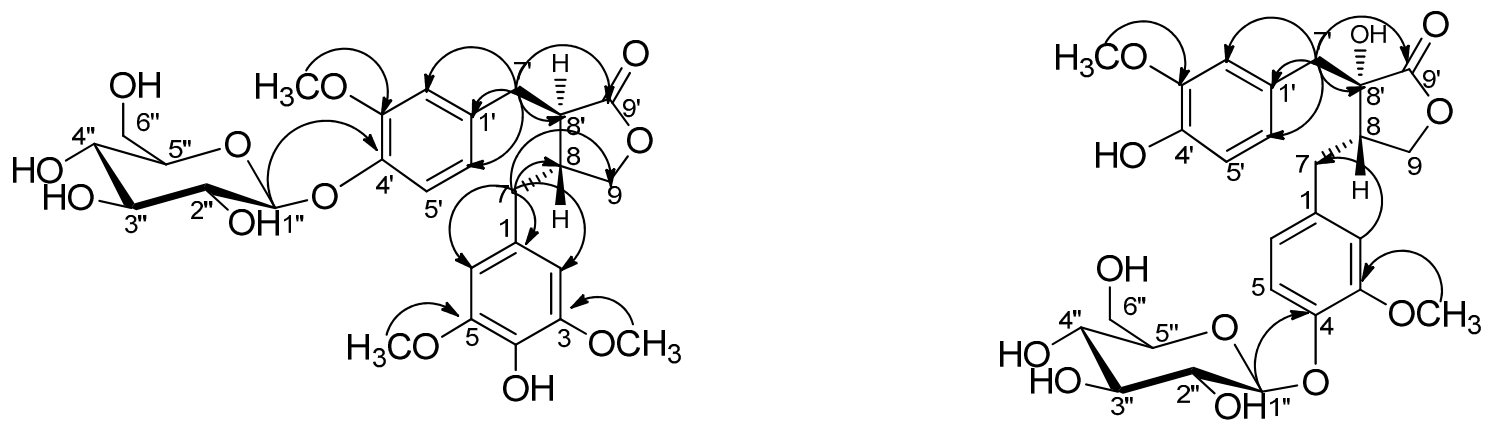
Compound 2 was isolated as white amorphous powder and its molecular formula was deduced as $\mathrm{C}_{26} \mathrm{H}_{32} \mathrm{O}_{12}$ from HRESIMS data which showed a $[\mathrm{M}+\mathrm{Na}]^{+}$ion at $\mathrm{m} / \mathrm{z} 559.1788$ (calcd. 559.1786). ${ }^{1} \mathrm{H}-\mathrm{NMR}$ and ${ }^{13} \mathrm{C}-\mathrm{NMR}$ spectral data (Table 1) suggested it was also a dibenzylbutyrolactone lignan compound. The presence of a diagnostic hydroxyl signal at $\delta_{\mathrm{H}} 6.21$ and an AB-type coupled protons at $\delta 2.98,2.83$ (each $1 \mathrm{H}, \mathrm{d}, J=13.8 \mathrm{~Hz}, \mathrm{H}-7^{\prime}$ ) in ${ }^{1} \mathrm{H}-\mathrm{NMR}$ spectrum suggested compound 2 was a 8 -hydroxydibenzylbutyrolactone lignan. ${ }^{1} \mathrm{H}$-NMR spectrum also displayed signals of two sets of ABX systems of the phenyl protons to present two 3,4-disubstituted phenyl groups. The presence of two methoxy groups at $\delta 3.70$ and 3.73 which had respective HMBC correlation with the carbon at $\delta 148.8$ (C-3) and carbon at $\delta 147.2\left(\mathrm{C}-3^{\prime}\right)$ as well as a phenolic hydroxy at $\delta 8.85\left(1 \mathrm{H}, \mathrm{s}, 4^{\prime}-\mathrm{OH}\right)$ suggested the aglycone of 2 was nortrachelogenin which was proved by HMBC correlations as shown in Figure 2. A sugar anomeric proton at $\delta 4.82$ (H-1") with coupling constant of $7.2 \mathrm{~Hz}$ in ${ }^{1} \mathrm{H}-\mathrm{NMR}$ spectrum and its corresponding spectral data in ${ }^{13} \mathrm{C}$-NMR spectrum indicated the presence of a $\beta$-D-glucopyranosyl group. HMBC correlation between the anomeric proton (H-1") and carbon at $\delta 145.1$ (C-4) or $\delta 145.4$ (C-4') implied that the glucopyranosyl group was connected to C-4 or C-4'. According to compound 2 having evident different NMR spectral data from that of nortracheloside which had been isolated from Caulis Trachelospermi in our previous research, it was determined that the location of $\beta$-D-glucopyranoside was at $\mathrm{C}-4$. Concerned the $\beta$ effect of 8 '-OH, optical isomers of 8 '-hydroxydibenzylbutyrolactone lignan produced different chemical shifts at C-7' and C-8. Trans isomers were at about $\delta 42.0$ and $\delta 43.7$, but $c i s$ isomers were at about $\delta 38.3$ and $\delta 48.1$. Moreover, $8 S$-configuration produced negative optical rotation in contrast with positive optical rotation of its epimer [19]. Compound 2 produced similar C-7' and C-8 signals at $\delta 40.0,42.7$ and negative optical rotation $[\alpha]_{D}^{25}-48.5(c 0.19, \mathrm{MeOH})$ revealed its absolute configuration was $8 S, 8^{\prime} S$. Compound 2 was accordingly assigned as nortrachelogenin 4-O- $\beta$-D-glucopyranoside, structurally $\left(8 S, 8^{\prime} S\right)$-4', $8^{\prime}$-dihydroxy-3,3'-dimethoxylignan-9,9'-olide-4- $O-\beta$-Dglucopyranoside. A publication of patent application has concerned the same planar structure of 2 by covering with common structures [20], but no data of the production (isolation or synthesis) and structure elucidation was provided. Therefore, we report herein $\mathbf{2}$ as a new compound.

The known compounds 3-8 were identified as matairesinol 4- $O-\beta$-D-glucopyranoside $(\mathbf{3})[21,22]$, nortrachelogenin 4,4'-di- $O$ - $\beta$-D-glucopyranoside (4) [23,24], 3,4,5-trimethoxyphenol 1-O- $\beta$-Dglucopyranoside (5) [25,26], kelampayoside A (6) [27], roseoside (7) [28] and tanegool (8) [29], respectively, by comparing spectroscopic data with literature values.

Initial investigation for new active herbal extract with inhibiting activity on JAK/STAT signaling pathway revealed that the extract of Caulis Trachelospermi, which was separated by $80 \%$ alcohol extraction and subsequent HP-20 macroporous resin column chromatography, was founded to strongly inhibit IFN- $\gamma$-induced STAT1-responsive luciferase activity (IFN- $\gamma / \mathrm{STAT} 1)$ with $\mathrm{IC}_{50}$ value of $2.43 \mu \mathrm{g} / \mathrm{mL}$ as well as inhibiting IL-6-induced STAT3-responsive luciferase activity (IL-6/STAT3) with IC $_{50}$ value of $1.38 \mu \mathrm{g} / \mathrm{mL}$ in vitro (Table 2). In subsquent screening for high active components from the extract, lignan compounds 1-4 together with other lignan compounds isolated in previous study [7-10] were first tested in concentration of $5 \mu \mathrm{M}$ which was close to the $\mathrm{IC}_{50}$ value of the extract. Following result showed aglycone compounds trachelogenin and arctigenin had high inhibition ratio more than 30\% (77.5\% and $32.6 \%$ ) on IFN- $\gamma / \mathrm{STAT} 1$ pathway as well as aglycone compounds trachelogenin, arctigenin and matairesinol inhibiting IL-6/STAT3 pathway with inhibition ratio of $96.1 \%, 44.2 \%$ and $89.8 \%$, respectively. Other aglycone compounds nortrachelogenin, 4-demethyltraxillagenin and 5-methoxytrachelogenin had low 
activity on IL-6/STAT3 pathway with inhibition ratio lower than 30\% (Table 3). The activities of trachelogenin, arctigenin and matairesinol were further confirmed by $\mathrm{IC}_{50}$ evaluation. $\mathrm{IC}_{50}$ values of trachelogenin and arctigenin on IFN- $\gamma / \mathrm{STAT} 1$ pathway were $3.14 \mu \mathrm{M}$ and $9.46 \mu \mathrm{M}$ as well as $\mathrm{IC}_{50}$ values of trachelogenin, arctigenin and matairesinol on IL-6/STAT3 pathway were $3.63 \mu \mathrm{M}, 6.47 \mu \mathrm{M}$ and $2.92 \mu \mathrm{M}$ (Table 2). Since signaling pathways are activated and deactivated within minutes after treatment. However, the luciferase assay in this study was performed after six hours of treatment. Therefore, the inhibition of the luciferase activity in this study may also be an indirect effect via the upregulation of the expression of other factors that then inhibit IFN- $\gamma /$ STAT1 and IL-6/STAT3 signaling pathways.

Table 2. $\mathrm{IC}_{50}$ values of the extract and active compounds from Caulis Trachelospermi on IFN- $\gamma /$ STAT1 and IL-6/STAT3 pathway.

\begin{tabular}{ccc}
\hline Sample & IFN- $\gamma /$ STAT1 & IL-6/STAT3 \\
\hline extract & $2.43 \mu \mathrm{g} / \mathrm{mL}$ & $1.38 \mu \mathrm{g} / \mathrm{mL}$ \\
trachelogenin & $3.14 \mu \mathrm{M}$ & $3.63 \mu \mathrm{M}$ \\
arctigenin & $9.46 \mu \mathrm{M}$ & $6.47 \mu \mathrm{M}$ \\
matairesinol & - & $2.92 \mu \mathrm{M}$ \\
Pyridone 6 $^{\text {a }}$ & $0.0049 \mu \mathrm{M}$ & - \\
AG490 $^{\text {a }}$ & - & $>100 \mu \mathrm{M}$ \\
\hline \multicolumn{3}{c}{${ }^{\text {a }}$ Positive control }
\end{tabular}

Table 3. Inhibition ratio (IR\%) values of lignan components from Caulis Trachelospermi on IFN- $\gamma /$ STAT1 and IL-6/STAT3 pathway.

\begin{tabular}{cccc}
\hline Compound & Conc. $(\boldsymbol{\mu M})$ & IFN- $\gamma /$ STAT1 & IL-6/STAT3 \\
\hline trachelogenin & 5 & $77.5 \%$ & $96.1 \%$ \\
tracheloside & 5 & $9.4 \%$ & $13.5 \%$ \\
trachelogenin 4'- $O$ - $\beta$-gentiobioside & 5 & $-8.0 \%$ & $-17.5 \%$ \\
nortrachelogenin & 5 & $-28.9 \%$ & $27.3 \%$ \\
nortracheloside & 5 & $-4.2 \%$ & $2.3 \%$ \\
nortrachelogenin 8'-O- $\beta$-D-glucoside & 5 & $-7.4 \%$ & $1.6 \%$ \\
nortrachelogenin 5'-C- $\beta$-D-glucoside & 5 & $-22.1 \%$ & $-9.3 \%$ \\
nortrachelogenin 4- $O-\beta$-D-glucopyranoside $(\mathbf{2})$ & 5 & $-3.5 \%$ & $10.9 \%$ \\
nortrachelogenin 4,4'-di- $O$ - $\beta$-D-glucopyranoside $(4)$ & 5 & $-2.7 \%$ & $-14.7 \%$ \\
arctigenin & 5 & $32.6 \%$ & $44.2 \%$ \\
arctiin & 5 & $15.4 \%$ & $-14.7 \%$ \\
matairesinol & 5 & $7.2 \%$ & $89.8 \%$ \\
matairesinoside & 5 & $-9.4 \%$ & $13.5 \%$ \\
matairesinol 4- $O-\beta$-D-glucopyranoside $(\mathbf{3})$ & 5 & $5.8 \%$ & $7.0 \%$ \\
matairesinol 4'- $O$ - $\beta$-gentiobioside & 5 & $-12.0 \%$ & $-12.9 \%$ \\
traxillagenin & 5 & $-1.9 \%$ & $-26.1 \%$ \\
traxillaside & 5 & $-5.1 \%$ & $-19.8 \%$ \\
4-demethyltraxillagenin & 5 & $7.1 \%$ & $22.4 \%$ \\
4-demethyltraxillaside $(\mathbf{1})$ & 5 & $6.3 \%$ & $-10.5 \%$ \\
5-methoxytrachelogenin & 5 & $-0.2 \%$ & $14.3 \%$ \\
5-methoxytracheloside & 5 & $-21.0 \%$ & $4.8 \%$ \\
\hline & & & \\
\hline & $5 \%$ & 5 &
\end{tabular}


Otherwise, all glycosyl or disaccharosyl compounds had no visible activities on IFN- $\gamma /$ STAT1 or IL-6/STAT3 pathway in concentration of $5 \mu \mathrm{M}$ (Table 3). In our previous research, trachelogenin and its glycoside tracheloside could be detected in rat plasma after oral administration of the extract of Caulis Trachelospermi [30]. Moreover, Nose et al. reported that tracheloside and arctiin could be converted to their aglycone trachelogenin and actigenin in rat large intestinal flora [31]. Therefore, the lignan glycosides may have effects in vivo on the JAK/STAT pathway via conversion to their aglycone in the intestinal tract.

\section{Experimental Section}

\subsection{General}

Optical rotations were obtained on an Optical Activity Limited polAAr 3005 spectropolarimeter. CD spectra were recorded on a Biologic M450 spectropolarimeter. 1D- and 2D-NMR experiment were performed on a JEOL JNM-GX 400 NMR spectrometer with TMS as internal standard. ESIMS and HRESIMS were conducted on an Applied Biosystems API 3000 LC-MS spectrometer and an Agilent 6520 Q-TOF LC-MS spectrometer, respectively. Semi-preparative RP-HPLC was carried out with Shimadzu instrument equipped with LC-15C pump and SPD-15C detector using an YMC $\mathrm{C}_{18}$ column $(5 \mu \mathrm{m}, 10 \times 250 \mathrm{~mm}$; YMC Co. Ltd., Kyoto, Japan). HP-20 macroporous resin (Mitsubishi Chemical Co., Tokyo, Japan), ODS (50 $\mu \mathrm{m}$, YMC Co. Ltd., Kyoto, Japan) and Sephadex ${ }^{\mathrm{TM}}$ LH-20 (GE Healthcare, Uppsala, Sweden) were employed for column chromatography (CC).

\subsection{Plant and Compound Materials}

Caulis Trachelospermi (the stems and leaves of T. jasminoides (Lindl.) Lem) were purchased from Beijing Qijing Chinese Herbs Factory, Beijing, in 2008 and authenticated by senior engineer Qiyun Ma, Beijing Institute of Pharmacology and Toxicology, Beijing, China. A voucher specimen (No. 20080130) has been deposited at the Department of Natural Products Chemistry, Beijing Institute of Pharmacology and Toxicology, Beijing, China. Compounds trachelogenin, tracheloside, trachelogenin 4'-O- $\beta$-gentiobioside, nortrachelogenin, nortracheloside, nortrachelogenin $\quad 8^{\prime}-O-\beta$-D-glucoside, nortrachelogenin 5'-C- $\beta$-D-glucoside, arctigenin, arctiin, matairesinol, matairesinoside, matairesinol 4'-O- $\beta$-gentiobioside, traxillagenin, traxillaside, 4-demethyltraxillagenin, 5-methoxytrachelogenin and 5-methoxytracheloside for the active tests were isolated and identified in our previous research [7-10].

\subsection{Extraction and Isolation}

Caulis Trachelospermi $(160 \mathrm{~kg}$ ) was extracted two times with $80 \%$ alcohol at boiling temperature. The extract was concentrated and diluted in $1600 \mathrm{~L} \mathrm{5 \%}$ alcohol. The solution was first centrifuged to remove the insoluble substance and then was passed through a HP-20 macroporous resin column (100 L) and eluted by $500 \mathrm{~L}$ water and $500 \mathrm{~L} 70 \%$ alcohol successively. The $70 \%$ alcohol elution was concentrated and dried to produce $5.3 \mathrm{~kg}$ product.

Guided by HPLC detection, the product (50 g) was applied on column chromatography over ODS eluted with $\mathrm{MeOH}-\mathrm{H}_{2} \mathrm{O}$ (4:6) to afford seven fractions (A-G). Fr-A was separated over ODS CC eluted with a gradient $10 \% \mathrm{MeOH}, 20 \% \mathrm{MeOH}, 30 \% \mathrm{MeOH}, 100 \% \mathrm{MeOH}$ and eight fractions were 
collected. Fr-A-3 was subjected to ODS CC eluted with $\mathrm{MeOH}-\mathrm{H}_{2} \mathrm{O}$ (15:85) and then purified by semi-preparative RP-HPLC with mobile phase $\mathrm{MeOH}-\mathrm{H}_{2} \mathrm{O}$ (16:84), to yield compounds 5 (11.4 mg) and $6(24.0 \mathrm{mg})$. Fr-A-4 was rechromatographed on an ODS column eluted with $\mathrm{MeOH}-\mathrm{H}_{2} \mathrm{O}(2: 8)$, followed by semi-preparative RP-HPLC [ $\mathrm{MeOH}-\mathrm{H}_{2} \mathrm{O}$ (17:83)] to give compound 8 (38.5 mg). Fr-A-5 was fractionated by ODS CC eluted with $\mathrm{MeOH}-\mathrm{H}_{2} \mathrm{O}(25: 75)$ and then purified by preparative RP-HPLC $\left[\mathrm{MeOH}-\mathrm{H}_{2} \mathrm{O}(17: 83)\right]$ to yield compound 4 (45.0 mg). Fr-A-6 was further isolated by ODS CC eluted with $\mathrm{MeOH}-\mathrm{H}_{2} \mathrm{O}(3: 7)$ then purified by Sephadex LH-20 (MeOH) and semi-preparative RP-HPLC $\left[\mathrm{MeOH}-\mathrm{H}_{2} \mathrm{O}(2: 8)\right]$ to afford compound 7 (1.9 mg). Fr-A-8 was chromatographed with identical method of Fr-A-6 to yield compound $2(8.0 \mathrm{mg})$. Fr-C was fractionated by ODS CC eluted with $\mathrm{MeOH}-\mathrm{H}_{2} \mathrm{O}$ (4:6) and seven fractions were obtained. Fr-C-4 was chromatographed over ODS CC $\left[\mathrm{MeOH}-\mathrm{H}_{2} \mathrm{O}(25: 75)\right]$ and purified by semi-preparative RP-HPLC [MeOH- $\mathrm{H}_{2} \mathrm{O}$ (28:72)] to afford compounds $\mathbf{1}(15.7 \mathrm{mg})$ and $\mathbf{3}(7.8 \mathrm{mg})$.

\subsection{Spectral Data}

4-Demethyltraxillaside (1). White amorphous powder. $[\alpha]_{D}^{25}-44.1(c 0.52, \mathrm{MeOH}) . \mathrm{CD}(\mathrm{MeOH}) \lambda_{\max } \mathrm{nm}$ $(\Delta \varepsilon): 233$ (-2.72), 276 (-0.58). ESIMS: $m / z 573[\mathrm{M}+\mathrm{Na}]^{+}$(Pos.), $549[\mathrm{M}-\mathrm{H}]^{-}$(Neg.). HRESIMS: $m / z$ : $573.1945[\mathrm{M}+\mathrm{Na}]^{+}$(Calcd. for $\left.\mathrm{C}_{27} \mathrm{H}_{34} \mathrm{O}_{12} \mathrm{Na}, 573.1942\right) .{ }^{1} \mathrm{H}-\mathrm{NMR}$ and ${ }^{13} \mathrm{C}-\mathrm{NMR}$ data see Table 1 .

Nortrachelogenin 4-O- $\beta$-D-glucopyranoside (2). White amorphous powder. $[\alpha]_{D}^{25}-48.5(c 0.19, \mathrm{MeOH})$. $\mathrm{CD}(\mathrm{MeOH}) \lambda_{\max } \mathrm{nm}(\Delta \varepsilon): 233(-1.94), 276(-0.28)$. ESIMS: $m / z 559[\mathrm{M}+\mathrm{Na}]^{+}$(Pos.), $535[\mathrm{M}-\mathrm{H}]^{-}$ (Neg.). HRESIMS: $m / z$ : $559.1788[\mathrm{M}+\mathrm{Na}]^{+}$(Calcd. for $\mathrm{C}_{26} \mathrm{H}_{32} \mathrm{O}_{12} \mathrm{Na}, 559.1786$ ). ${ }^{1} \mathrm{H}-\mathrm{NMR}$ and ${ }^{13} \mathrm{C}-\mathrm{NMR}$ data see Table 1.

Matairesinol 4-O- $\beta$-D-glucopyranoside (3). White amorphous powder. $[\alpha]_{D}^{25}-34.0$ (c 0.40, MeOH). $\mathrm{CD}(\mathrm{MeOH}) \lambda_{\max } \mathrm{nm}(\Delta \varepsilon): 233(-2.23), 276(-0.39)$. ESIMS: $m / z 543[\mathrm{M}+\mathrm{Na}]^{+}$(Pos.), $519[\mathrm{M}-\mathrm{H}]^{-}$ (Neg.). HRESIMS: $m / z$ : $543.1838[\mathrm{M}+\mathrm{Na}]^{+}$(Calcd. for $\left.\mathrm{C}_{26} \mathrm{H}_{32} \mathrm{O}_{11} \mathrm{Na}, 543.1837\right) .{ }^{1} \mathrm{H}-\mathrm{NMR}$ (DMSO- $d_{6}$ ): $\delta_{\mathrm{H}} 6.67(1 \mathrm{H}, \mathrm{d}, J=1.8 \mathrm{~Hz}, \mathrm{H}-2), 6.96(1 \mathrm{H}, \mathrm{d}, J=8.3 \mathrm{~Hz}, \mathrm{H}-5), 6.57(1 \mathrm{H}, \mathrm{dd}, J=8.3,1.8 \mathrm{~Hz}, \mathrm{H}-6)$, 2.44-2.48 (3H, m, H-7, 8), 4.05 (1H, m, Ha-9), 3.87 (1H, dd, $J=11.2,4.8 \mathrm{~Hz}, \mathrm{Hb}-9), 6.76$ (1H, d, $\left.J=1.8 \mathrm{~Hz}, \mathrm{H}-2^{\prime}\right), 6.69\left(1 \mathrm{H}, \mathrm{d}, J=8.0 \mathrm{~Hz}, \mathrm{H}-5^{\prime}\right), 6.60\left(1 \mathrm{H}, \mathrm{dd}, J=8.0,1.8 \mathrm{~Hz}, \mathrm{H}-6{ }^{\prime}\right), 2.83$ (1H, dd, $\left.J=13.5,5.1 \mathrm{~Hz}, \mathrm{Ha}-7^{\prime}\right), 2.73\left(1 \mathrm{H}, \mathrm{m}, \mathrm{Hb}-7^{\prime}\right), 2.69\left(1 \mathrm{H}, \mathrm{m}, \mathrm{H}-8^{\prime}\right), 8.84\left(1 \mathrm{H}, \mathrm{s}, 4^{\prime}-\mathrm{OH}\right), 3.72(6 \mathrm{H}, \mathrm{s}$, 3,3'-OMe), $4.82(1 \mathrm{H}, \mathrm{d}, J=7.4 \mathrm{~Hz}, \mathrm{H}-1 ") .{ }^{13} \mathrm{C}-\mathrm{NMR}$ (DMSO- $\left.d_{6}\right)$ : $\delta \mathrm{c} 132.6(\mathrm{C}-1), 112.9$ (C-2), 148.8 (C-3), 145.1 (C-4), 115.3 (C-5), 120.5 (C-6), 36.9 (C-7), 40.9 (C-8), 70.8 (C-9), 129.0 (C-1'), 113.5 (C-2'), 147.5 (C-3'), 145.1 (C-4'), $115.4\left(\mathrm{C}-5^{\prime}\right), 121.6$ (C-6'), 33.8 (C-7'), 45.7 (C-8'), 178.6 (C-9'), 100.2 (C-1"), 73.3 (C-2"), 77.1 (C-3"), 69.7 (C-4"), 76.9 (C-5"), 60.7 (C-6"), 55.6 (C-3, 3'-OMe).

Nortrachelogenin 4,4'-di-O- $\beta$-D-glucopyranoside (4). White amorphous powder. ESIMS: $\mathrm{m} / z 716$ $\left[\mathrm{M}+\mathrm{NH}_{4}\right]^{+}, 721[\mathrm{M}+\mathrm{Na}]^{+}$(Pos.), $697[\mathrm{M}-\mathrm{H}]^{-}$(Neg.). ${ }^{1} \mathrm{H}-\mathrm{NMR}\left(\mathrm{DMSO}-d_{6}\right): \delta_{\mathrm{H}} 6.72(1 \mathrm{H}, \mathrm{d}, J=1.7 \mathrm{~Hz}$, H-2), 6.99 (1H, d, $J=8.5 \mathrm{~Hz}, \mathrm{H}-5), 6.74(1 \mathrm{H}, \mathrm{dd}, J=8.5,1.7 \mathrm{~Hz}, \mathrm{H}-6), 2.65$ (1H, m, Ha-7), 2.46 (br d, $J=10.1 \mathrm{~Hz}, \mathrm{Hb}-7), 2.40$ (1H, m, H-8), 3.97 (2H, br d, $J=8.3 \mathrm{~Hz}, \mathrm{H}-9), 6.83$ (1H, d, $J=1.6 \mathrm{~Hz}, \mathrm{H}-2$ '), $6.97\left(1 \mathrm{H}, \mathrm{d}, J=8.3 \mathrm{~Hz}, \mathrm{H}-5^{\prime}\right), 6.62\left(1 \mathrm{H}, \mathrm{dd}, J=8.3,1.6 \mathrm{~Hz}, \mathrm{H}-6{ }^{\prime}\right), 3.02$ (1H, d, $\left.J=13.5 \mathrm{~Hz}, \mathrm{Ha}-7^{\prime}\right)$, $2.88\left(1 \mathrm{H}, \mathrm{d}, J=13.5 \mathrm{~Hz}, \mathrm{Hb}-7^{\prime}\right), 6.30$ (1H, s, 8'-OH), 3.70, 3.73 (each 3H, s, 3, 3'-OMe), 4.85, 4.82 (each 1H, d, $\left.J=7.5 \mathrm{~Hz}, \mathrm{H}-1 ", \mathrm{H}-1{ }^{\prime \prime \prime}\right) .{ }^{13} \mathrm{C}-\mathrm{NMR}$ (DMSO- $d_{6}$ ): $\delta \mathrm{c} 132.9$ (C-1), 129.2 (C-1'), 113.0 (C-2), 
114.8 (C-2'), 148.4, 148.8 (C-3, 3'), 145.1, 145.5 (C-4, 4'), 115.3 (C-5, 5'), 120.5 (C-6), 122.5 (C-6'), 30.9 (C-7), 42.8 (C-8), 70.0 (C-9), 75.4 (C-8'), 178.0 (C-9'), 100.2, 100.0 (C-1", 1"'), 73.2 (C-2", 2"'), 77.0 (C-3", 3"'), 69.7 (C-4", 4"'), 76.9 (C-5", 5"'), 60.6, 60.7 (C-6", 6"'), 55.6, 55.7 (C-3, 3'-OMe).

\subsection{Cell Lines and Reagents}

HepG2/STAT1 (HepG2/STAT3) cells, a gift from Prof. Xinyuan Fu (National University of Singapore, Singapore, Singapore), were a human hepatoma derived cell line cells stably transfected with a STAT1 (STAT3)-responsive firefly luciferase reporter plasmid. Luciferase assay kit was purchased from Promega Corp., Madison, WI, USA. Recombinant human IFN- $\gamma$ was purchased from Shanghai Clone High Biological Technology Co Ltd., Shanghai, China. Recombinant human IL-6 was purchased from Pepprotech Inc., Rocky Hill, NJ, USA. Pyridone 6 and AG490 were purchased from Merck Biosciences, Darmstadt, Germany and Calbiochem, San Diego, CA, USA, respectively.

\subsection{Luciferase Assay}

Luciferase assay was performed as previous described [32]. HepG2/STAT1 (HepG2/STAT3) cells $\left(2 \times 10^{4}\right.$ per well) maintained in a DMEM medium were seeded into 96-well cell culture microplates and allowed to grow for $24 \mathrm{~h}$ at $37{ }^{\circ} \mathrm{C}$ in a $5 \% \mathrm{CO}_{2}$ incubator, and then treated with test samples for $1 \mathrm{~h}$ followed by stimulation with $50 \mathrm{IU} / \mathrm{mL} \mathrm{IFN}-\gamma(10 \mathrm{ng} / \mathrm{mL}$ IL-6) for $6 \mathrm{~h}$. Luciferase activity was determined using the Promega luciferase kits according to the manufacturer's instruction. The cell number was counted at seeding and controlled by equal seeding. All luciferase assays were repeated at least twice to minimize the variance caused by cell number. IFN- $\gamma$ (IL-6)-induced STAT1 (STAT3)-responsive luciferase activity (\% of control) was calculated as $\{$ [Fluorescence intensity with sample and IFN $-\gamma$ (IL-6) treatment - Fluorescence intensity without IFN- $\gamma$ (IL-6) treatment]/[Fluorescence intensity with IFN- $\gamma($ IL-6) treatment - Fluorescence intensity without IFN- $\gamma($ IL-6) treatment] $\} \times 100$.

\section{Conclusions}

In summary, two new dibenzylbutyrolactone lignans, 4-demethyltraxillaside (1) and nortrachelogenin 4- $O-\beta$-D-glucopyranoside (2), together with six known compounds were isolated from Caulis Trachelospermi. Their structures and stereochemistries were established by the analysis of 1D, 2D-NMR, MS, OR and CD spectra. The extract of Caulis Trachelospermi and its main dibenzylbutyrolactone lignan components had strong inhibiting activity on JAK/STAT pathway, and it may be an important mechanism for the reported anticancer and anti-inflammation function of Caulis Trachelospermi. However, it is still unclear whether the inhibition of IFN- $\gamma /$ STAT1 and IL-6/STAT3 signaling pathways by the active components is direct on these pathways or whether it occurs via the upregulation of the expression of other factors that then inhibit these signaling pathways. Further research is needed for the determination of their precise target.

\section{Supplementary Materials}

Supplementary materials can be accessed at: http://www.mdpi.com/1420-3049/19/8/11560/s1. 


\section{Acknowledgments}

This work was supported by the National Science and Technology Major Project of China (No. 2013ZX09102-018 and 2012ZX09301003-001-004) and the project of Beijing Municipal Science \& Technology Commission (Z131100006513013). Bioactive assays by Xiao Yu and Qing Zhang, Shanghai Institute of Materia Medica, Chinese Academy of Sciences are acknowledged.

\section{Author Contributions}

Xiao-Ting Liu achieved the whole experimental work, including isolation, purification, identification and prepared the manuscript. Neng-Jiang Yu was the project leader who took charge of the throughout research and accomplished writing of the paper. Zhe-Xing Wang, Yu Yang and Yi-Min Zhao contributed to this work by coaching and assisting Liu's experiments. Lin Wang and Ruo-Feng Sun took part in the extraction and isolation.

\section{Conflicts of Interest}

The authors declare no conflict of interest.

\section{References}

1. Committee of Pharmacopoeia of the People's Republic of China. Pharmacopoeia of the People's Republic of China (First Part); China Medical Science Technology Press: Beijing, China, 2010; p. 252.

2. Nishibe, S.; Han, Y.M. Chemical constituents from Trachelosperomum jasminoides and its anticancer activity. World Phytomed. 2002, 17, 57-58.

3. Lee, M.H.; Lee, J.M.; Jun, S.H.; Ha, C.G.; Lee, S.-H.; Kim, N.M.; Lee, J.H.; Ko, N.Y.; Mun, S.H.; Park, S.H.; et al. In vitro and in vivo anti-inflammatory action of the ethanol extract of Trachelospermi caulis. J. Pharm. Pharmacol. 2007, 59, 123-130.

4. Tan, X.Q.; Chen, H.S.; Liu, R.H.; Tan, C.H.; Xu, C.L.; Xuan, W.D.; Zhang, W.D. Lignans from Trachelospermum jasminoides. Planta Med. 2005, 71, 93-95.

5. Tan, X.Q.; Chen, H.S.; Zhou, M.; Zhang, Y. Triterpenoids from canes with leaves of Trachelospermum jasminoides. Chin. Tradit. Herb. Drugs 2006, 37, 171-174.

6. Tan, X.Q.; Guo, L.J.; Chen, H.S.; Wu, L.S.; Kong, F.F. Study on the flavonoids constituents of Trachelospermum jasminoides. J. Chin. Med. Mater. 2010, 33, 58-60.

7. Yu, N.J.; Zhao, Y.M.; Ren, F.X. Total Lignans Extract from Caulis Trachelospermi, Its Extraction Method, and the Medicinal Usage of the Extract and Its Active Constituents. CN 200510093357.X, 26 August 2005.

8. Zhu, C.C.; Jing, L.; Yu, N.J.; Yang, X.D.; Zhao, Y.M. A new lignan and active compounds inhibiting NF- $\kappa \mathrm{B}$ signaling pathway from Caulis Trachelospermi. Acta Pharm. Sin. B 2013, 3, 109-112.

9. Jing, L.; Yu, N.J.; Zhao, Y.M.; Li, Y.S. Trace chemical constituents contained in Trachelospermum jasminoides and structure identification. China J. Chin. Mater. Med. 2012, 37, $1581-1585$. 
10. Yuan, Q.S.; Yu, N.J.; Zhao, Y.M.; Xu, B.; Yao, Z.W. Chemical constituents from Trachelospermum jasminoides. Chin. Tradit. Herb. Drugs 2010, 41, 179-181.

11. Jing, L.; Yu, N.J.; Li, Y.S.; Fu, L.; Zhao, Y.M. Novel lignans from the stems and leaves of Trachelospermum jasminoides. Chin. Chem. Lett. 2011, 22, 1075-1077.

12. Kisseleva, T.; Bhattacharya, S.; Braunstein, J.; Schindler, C.W. Signaling through the JAK/STAT pathway, recent advances and future challenges. Gene 2002, 285, 1-24.

13. Buettner, R.; Mora, L.B.; Jove, R. Activated STAT signaling in human tumors provides novel molecular targets for therapeutic intervention. Clin. Cancer Res. 2002, 8, 945-954.

14. Al Siddiquee, Z.K.; Turkson, J. STAT3 as a target for inducing apoptosis in solid and hematological tumors. Cell Res. 2008, 18, 254-267.

15. De Hooge, A.S.; van de Loo, F.A.; Koenders, M.I.; Bennink, M.B.; Arntz, Q.J.; Kolbe, T.; van den Berg, W.B. Local activation of STAT-1 and STAT-3 in the inflamed synovium during zymosan-induced arthritis. Arthritis Rheum. 2004, 50, 2014-2023.

16. Liu, Y.Q.; Yu, N.J.; Yang, X.D.; Zhao, Y.M. Study on HPLC fingerprint of Trachelospermum jasminoides. China J. Chin. Mater. Med. 2009, 34, 727-730.

17. Agrawal, P.K. NMR spectroscopy in the structural elucidation of oligosaccharides and glycosides. Phytochemistry 1992, 31, 3307-3330.

18. Gu, L.H.; Wang, S.X.; Li, X.; Zhu, T.R. Studies on antibacterial constituents from Gerbenra anandria (L.) Sch. Bip. Acta Pharmacol. Sin. 1987, 22, 272-277.

19. Khamlach, K.; Dhal, R.; Brown, E. Total syntheses of (-)-trachelogenin, (-)-nortrachelogenin and (+)-wikstromol. Tetrahedron Lett. 1989, 30, 2221-2224.

20. Peuhu, E.; Eriksson, J.; Holmbom, T.; Eklund, P.; Sjoholm, R. Pharmaceutical compositions comprising 8-substituted dibenzylbutyrolactone lignans. U.S. Patent 2013/0281381 A1, 24 October 2013.

21. Palter, R.; Lundin, R.E. A bitter principle of safflower; Matairesinol monoglucoside. Phytochemistry 1970, 9, 2407-2409.

22. Palter, R.; Haddon, W.F.; Lundin, R.E. The complete structure of matairesinol monoglugoside. Phytochemistry 1971, 10, 1587-1589.

23. Nishibe, S.; Hisada, S.; Inagaki, I. Lignan diglucosides from Trachelospermum asiaticum. Phytochemistry 1972, 11, 3084-3085.

24. Nishibe, S.; Hisada, S.; Inagaki, I. Lignans of Trachelospermum asiaticum var. intermedium. V. Isolation of nortrachelogenin-4,4'-di-O- $\beta$-D-glucopyranoside. Chem. Pharm. Bull. 1973, 21, 1114-1117.

25. Steinbeck, C.; Schneider, C.; Rotscheidt, K.; Breitmaier, E. A 4-methy-7-hydroxyphthalide glycoside and other constituents from Quillaja saponaria molina. Phytochemistry 1995, 40, 1313-1315.

26. Verotta, L.; Dell'Agli, M.; Giolito, A.; Guerrini, M.; Cabalion, P.; Bosisio, E. In vitro antiplasmodial activity of extracts of Tristaniopsis species and identification of the active constituents: Ellagic acid and 3,4,5-trimethoxyphenyl-(6'-O-galloyl)- $O$ - $\beta$-D-glucopyranoside. J. Nat. Prod. 2001, 64, 603-607.

27. Kanchanapoom, T.; Kasai, R.; Yamasaki, K. Iridoid and phenolic glycosides from Morinda coreia. Phytochemistry 2002, 59, 551-556. 
28. Zhang, J.M.; Shi, X.F.; Ma, Q.H.; He, F.J.; Fan, B.; Wang, D.D.; Liu, D.Y. Chemical constituents from pine needles of Cedrus deodara. Chem. Nat. Compd. 2011, 47, 272-274.

29. Abe, F.; Yamauchi, T. Tanegoside A, B and C, lignan glycosides from Trachelospermum liukiuense. Chem. Pharm. Bull. 1990, 38, 2143-2145.

30. Li, L.; Meng, F.H.; Guo, J.F.; Sun, L.; Yu, N.J.; Zhao, Y.M. Simultaneous quantification of tracheloside and trachelogenin in rat plasma using liquid chromatography/tandem mass spectrometry. J. Chromatogr. B Analyt. Technol. Biomed. Life Sci. 2011, 879, 1033-1037.

31. Nose, M.; Fujimoto, T.; Takeda, T.; Nishibe, S.; Ogihara, Y. Structural transformation of lignan compounds in rat gastrointestinal tract. Planta Med. 1992, 58, 520-523.

32. Wang, Y.; Ma, X.Q.; Yan, S.S.; Shen, S.S.; Zhu, H.L.; Gu, Y.; Wang, H.B.; Qin, G.W.; Yu, Q. 17-Hydroxy-jolkinolide B inhibits signal transducers and activators of transcription 3 signaling by covalently cross-linking Janus Kinases and induces apoptosis of human cancer cells. Cancer Res. 2009, 69, 7302-7310.

Sample Availability: Not available.

(C) 2014 by the authors; licensee MDPI, Basel, Switzerland. This article is an open access article distributed under the terms and conditions of the Creative Commons Attribution license (http://creativecommons.org/licenses/by/3.0/). 